\title{
Interacción genotipo por ambiente de siete variedades de papa en la zona papera de Chiriquí, Panamá
}

\author{
A.Gutiérrez-Gutiérrez ${ }^{1}$, J. Muñoz ${ }^{1}$
}

\begin{abstract}
RESUMEN
El consumo de papa procesada, la cual proviene del extranjero en su totalidad, ha aumentado en Panamá en las últimas décadas mientras que la producción nacional ha registrado un ligero descenso. En el país es reducida la oferta de variedades de papa para consumo fresco y no existen variedades con aptitud para procesamiento. El estudio se realizó en el corregimiento de Cerro Punta, provincia de Chiriquí, entre diciembre de 2005 y diciembre de 2007 y tuvo como objetivo seleccionar variedades de papa con alto potencial de rendimiento, tolerancia a las principales plagas y aptitud para procesamiento y/o consumo fresco. Se evaluaron seis variedades de papa generados por el Instituto de Investigaciones Agropecuarias (INIA) de Chile. Como control se utilizó la variedad Granola. Los datos fueron procesados utilizando el modelo AMMI (Additive Main Effects and Multiplicative Interaction), evidenciando un comportamiento diferencial entre los genotipos. Las variedades Pehuenche-INIA y Karú-INIA presentaron el mayor rendimiento y estabilidad, con alto valor del Componente Principal 1 (PCA1) y valores de Componente Principal 2 (PCA2) más cercanos a cero. Pehuenche-INIA presentó un alto contenido de sólidos en los tubérculos, siendo los mismos de un tamaño menor al promedio, mientras que Karu-INIA y R-91015-20 sobresalen por un mayor tamaño de tubérculos.
\end{abstract}

Palabras clave: rendimiento, adaptabilidad, estabilidad.

\section{Genotype-environment interaction of seven potato cultivars in the high lands of Chiriqui, Panama.}

\begin{abstract}
The consumption of processed potato in Panama, which comes from abroad in its entirety, has increased in recent decades, while domestic production has shown a slight decrease. At the national level there is a reduced supply of potato varieties for fresh consumption, and there are no apt-for-processing varieties. Aimed at selecting potato cultivars with high yield potential, tolerance to major pests and suitability for processing and / or fresh consumption, the present study was conducted in the village of Cerro Punta of the Province of Chiriqui, from December 2005 to December 2007. Six potato cultivars generated by the Institute of Agricultural Research (INIA) of Chile were evaluated. German variety 'Granola' was used as control check. The data were processed using an AMMI (Additive Main Effects and Multiplicative Interaction) model, which showed differential behaviors among genotypes. Varieties 'Pehuenche-INIA' and 'Karu-INIA' showed the best performance and stability, reaching high Principal Component 1 (PCA1) values and the closest to zero Principal Component 2 (PCA2) values. Pehuenche-INIA was observed to present high solid content in smaller-than-the-average tubers, while Karu-INIA and R-91015-20 stand out for bearing larger tubers.
\end{abstract}

Key words: performance, adaptability, stability.

\footnotetext{
${ }^{1}$ Estación Experimental de Cerro Punta. Instituto de Investigación Agropecuaria de Panamá (IDIAP) Correo electrónico: arnulfogutierrezg@yahoo.es
} 


\section{Introducción}

La variedad de papa (Solanum tuberosum L.) Granola, perteneciente a la subespecie tuberosum, sostiene más del $90 \%$ de la actividad papera en Panamá. La misma se caracteriza por un alto potencial de rendimiento; sin embargo, presenta bajos niveles de tolerancia a plagas importantes del cultivo y un contenido de sólidos insuficiente para ser procesada industrialmente.

La presencia de una alta presión de plagas como el tizón tardío (Phytophthora infestans), el Nemátodo del Quiste de la Papa (NQP; Globodera spp.) y otras como la Dormidera (Ralstonia solanacearum), la Pata negra (Pectobacterium carotovorum), la mosca minadora (Liriomyza spp.) y la polilla de la papa (Tecia solanivora) obliga a los productores a tomar constantes medidas de control, usualmente químicas, de alto costo y no siempre efectivas, generando interrogantes en torno a la seguridad ambiental y la salud pública, particularmente en lo concerniente a la acumulación de residuos de ingredientes activos en los tubérculos destinados para el consumo humano. En la práctica agrícola, constantemente emergen nuevos patógenos o los conocidos desarrollan resistencia o tolerancia a plaguicidas en uso. Todo lo anterior mantiene en un alto nivel de riesgo la producción de papa en el país.

Por otro lado, el consumo de papa en Panamá ha aumentado en las últimas décadas mientras que la producción nacional ha registrado un ligero descenso, sin embargo, las importaciones han crecido sostenidamente (MIDA, 2006). El aumento más significativo en el consumo ha sido de papa procesada, lo que obedece a cambios en los hábitos de consumo de la población que prefiere productos semielaborados que no requieren de mucho tiempo y esfuerzo al momento de ser preparados para consumo; el $100 \%$ de esa demanda es cubierta por importaciones. La principal causa de esta situación que limita las posibilidades de crecimiento del cultivo y que tiene en desventaja a los productores nacionales, es que no se cuenta con variedades de papa con un contenido de sólidos en los tubérculos mínimo de 18-19\%, que se requiere para procesamiento (CIP, 2007).
En este escenario cobra especial vigencia, como proceso continuo y necesario, la búsqueda de variedades alternativas, con alto potencial de rendimiento y mejores niveles de resistencia o tolerancia a las principales plagas que afectan el cultivo. Además, los factores mencionados deben estar conjugados, en el genotipo, con las respectivas características de calidad que garanticen la aceptación del producto por el consumidor (forma, color, tipo de piel y ojos de los tubérculos).

En Panamá, el mejoramiento genético de la papa se ha limitado a la evaluación de la adaptabilidad local de variedades generadas en otras latitudes. En este sentido, el principal aspecto a tener en consideración es el efecto de la interacción genotipo-ambiente (Bradshaw y MacKay, 1994; Yue et al., 1997; Scapim et al., 2000; Contreras y Krarup, 2000), es decir, observar los efectos del ambiente local sobre la expresión fenotípica de los genotipos. El contenido de sólidos en los tubérculos, por ejemplo, es una característica heredable, pero que se ve influenciada por las condiciones agroecológicas (Dale y MacKay, 1994; Kumlay et al., 2002; Westermann et al., 1994). Durante los últimos años, efectivamente, se han recomendado variedades que se adaptan a las condiciones de las tierras altas de Chiriquí, algunas de las cuales se han mantenido por mucho tiempo y han permitido a la papa mantenerse como el principal rubro de esta zona del país (IDIAP, 1984).

La oferta de variedades de papa a nivel mundial es amplia, sin embargo por lo limitado del mercado panameño, el desarrollo de nuevas variedades no representa interés para las grandes empresas obtentoras que, antes de comercializar las mismas, deben pasar por un proceso de evaluaciones oficiales in situ. Surge, entonces la necesidad de apoyar a los productores nacionales en la búsqueda de nuevas variedades. Con ese enfoque se ha establecido una alianza entre el Instituto de Investigación Agropecuaria de Panamá (IDIAP), productores de papa y el Instituto de Investigaciones Agropecuarias (INIA) de Chile, lo que permitió la realización del presente estudio, cuyo objetivo fue la evaluación de la adaptabilidad y estabilidad del 
rendimiento, la calidad de la producción y otras características de clones y variedades de papa generadas por el INIA de Chile, en la búsqueda de variedades alternativos para los productores de papa en Panamá.

\section{Materiales y métodos}

En este estudio se incluyeron seis variedades (Karu-INIA, Pehuenche-INIA, Ona-INIA， R91015-20, R-89054-3 y R-91193-1) que presentaron características morfológicas del tubérculo aceptables para consumidores; también se incluyó la variedad Granola para efectos comparativos. La investigación se realizó entre 2005 y 2007, en siete sitios o localidades (fincas de productores), con microclimas diferenciados considerados como ambientes en este estudio ubicadas en el Corregimiento de Cerro Punta, principal área papera de Panamá. Aquí se registran temperaturas máximas de $21,2{ }^{\circ} \mathrm{C}$ y mínimas de $7,6^{\circ} \mathrm{C}$, precipitaciones promedio mensual y anual de 196,23 y de $2353,97 \mathrm{~mm}$, respectivamente y un promedio mensual de humedad relativa de $80 \%$.
Los suelos del área, de origen volcánico, corresponden al orden Andisol caracterizados por su alta fertilidad. La ubicación geográfica, año, temporada y altitud de los sitios de los ensayos se detallan en la Tabla 1.

El manejo del cultivo fue realizado por los productores. En cada localidad, las variedades (tratamientos) fueron dispuestas mediante un diseño de bloques completos al azar con tres repeticiones. La unidad experimental consistió de cuatro surcos de $5 \mathrm{~m}$ de largo, separados entre sí a $0,75 \mathrm{~m}$ y como parcela efectiva se tomaron los dos surcos centrales. Se evaluó el peso promedio de los tubérculos y el rendimiento total (todos los tubérculos con un diámetro superior a los $35 \mathrm{~mm}$ ), transformados a toneladas por hectárea $\left(\mathrm{t} \cdot \mathrm{ha}^{-1}\right)$. Se realizó el análisis de varianza combinado para el peso promedio de los tubérculos y se calculó el contenido de sólidos totales en los tubérculos mediante el uso de un hidrómetro, según metodología propuesta por el Centro Internacional de la Papa (CIP, 2007).

Tabla1. Características de los ambientes (sitios de los ensayos)

\begin{tabular}{|c|c|c|c|c|}
\hline Localidad & Ciclo & Temporada & $\begin{array}{l}\text { Altitud } \\
\text { msnm }\end{array}$ & Ubicación \\
\hline Alto Las Nubes & 2007 & Lluvias & 2010 & $8^{\circ} 52^{\prime} 716^{\prime \prime} \mathrm{N} \quad 82^{\circ} 35^{\prime} 58.6^{\prime \prime} \mathrm{W}$ \\
\hline Las Nubes & 2007 & Lluvias & 2034 & $8^{\circ} 53^{\prime} 105^{\prime \prime} \mathrm{N} \quad 82^{\circ} 35^{\prime} 968^{\prime \prime} \mathrm{W}$ \\
\hline La Garita & 2007 & Lluvias & 1965 & $8^{\circ} 52^{\prime} 256^{\prime \prime} \mathrm{N} \quad 82^{\circ} 36^{\prime} 492^{\prime \prime} \mathrm{W}$ \\
\hline Alto Tribaldos & 2007 & Lluvias & 1969 & $8^{\circ} 49^{\prime} 761^{\prime \prime} \mathrm{N} \quad 82^{\circ} 35^{\prime} 816^{\prime \prime} \mathrm{W}$ \\
\hline $\begin{array}{l}\text { Cerro Punta (Anef, } \\
\text { invierno) }\end{array}$ & 2006 & Lluvias & 1914 & $8^{\circ} 51^{\prime} 32.7^{\prime \prime} \mathrm{N} 82^{\circ} 34^{\prime} 43.6^{\prime \prime} \mathrm{W}$ \\
\hline Cerro Punta (Anef, verano) & 2005 & Seca & 1912 & $8^{\circ} 51^{\prime} 43.6^{\prime \prime} \mathrm{N} 82^{\circ} 34^{\prime} 47.7^{\prime \prime} \mathrm{W}$ \\
\hline Alto Pineda & 2006 & Lluvias & 2160 & $82^{\circ} 34^{\prime} 26.8^{\prime \prime} \mathrm{W}$ \\
\hline
\end{tabular}

Para los datos del rendimiento, se llevó a cabo un análisis de varianza por localidad y el combinado para el conjunto, como paso previo al análisis de la estabilidad según el modelo AMMI (Additive Main Effects and Multiplicative Interaction). En este caso no se realizó la prueba de homogeneidad de la varianza del error ya que no es restrictivo para este modelo, que genera los componentes principales (PC) tomando en cuenta las medias de cada genotipo en las localidades evaluadas sin considerar las repeticiones (Crossa, 1990; Zobel et al., 1988). Para el análisis combinado se consideró a los genotipos como un efecto fijo y a los ambientes como efecto aleatorio, utilizándose los procedimientos PROC GLM del programa SAS. 
El modelo estadístico, del análisis combinado de varianza utilizado correspondió a:

$$
Y_{i j k}=\mu+g_{i}+a_{j}+(g a)_{i j}+r_{j k}+e_{i j k}
$$

Donde:

$$
\begin{aligned}
\mathrm{Y}_{\mathrm{ge}}= & \text { Rendimiento promedio del } \\
& \text { genotipo en la repetición del } \\
& \text { ambiente, } \\
\mu= & \text { Rendimiento promedio de los } \\
& \text { genotipos, } \\
\mathrm{g}_{\mathrm{i}}= & \text { Efecto del genotipo, } \\
\mathrm{a}_{\mathrm{j}}= & \text { Efecto del ambiente, } \\
(\mathrm{ga})_{\mathrm{ij}}= & \text { Efecto de la interacción genotipo } \mathrm{x} \\
& \text { ambiente, } \\
\mathrm{r}_{\mathrm{jk}}= & \text { Efecto de la repetición en el } \\
& \text { ambiente, } \\
\mathrm{e}_{\mathrm{ij}}= & \text { Efecto aleatorio del error } \\
& \text { experimental }
\end{aligned}
$$

Una vez detectada la presencia de la interacción genotipo x localidad, se procedió al análisis de estabilidad utilizando el modelo AMMI y el grafico Biplot GGE (Vargas y Crossa, 2000; Cornelius et al., 1996; Crossa and Cornelius, 1997).

$$
\mathrm{Y}_{\mathrm{ge}}=\mu+\alpha_{\mathrm{g}}+\beta_{\mathrm{e}}+\Sigma^{\mathrm{N}} \lambda_{\mathrm{n}} \mathrm{Y}_{\mathrm{gn}} \delta_{\mathrm{en}}+\rho_{\mathrm{ge}}
$$

Donde:

$\mathrm{Y}_{\mathrm{ijk}}=$ Rendimiento de la variedad $\mathrm{g}$ en un ambiente e,

$\mu=$ Media general

$\alpha_{\mathrm{g}}=$ Efecto de las desviaciones de las medias de las variedades,

$\beta_{\mathrm{e}}=$ Efecto de las desviaciones de las medias de los ambientes,

$\Sigma^{\mathrm{N}}=$ Número de PCA retenidos en el modelo,

$\lambda_{\mathrm{n}}=$ Valor singular para el PCA,

$\mathrm{Y}_{\mathrm{gn}}=$ Valores de los vectores de los genotipos

$\delta_{\text {en }}=$ Valores de los vectores para cada ambiente

$\rho_{\mathrm{ge}}=$ Residual

\section{Resultados y discusión}

El análisis de varianza combinado para la variable peso promedio, en gramos (tamaño de los tubérculos), detectó diferencias significativas $(\mathrm{p}<0,01)$ para las variedades evaluadas (CV 20,76\%), destacando Karú-INIA y R-91015-20 con un peso promedio significativamente superior. La variedad Pehuenche-INIA, produjo los tubérculos de menor tamaño, pero no se diferenció estadísticamente de R-91193-1 y OnaINIA (Figura 1).

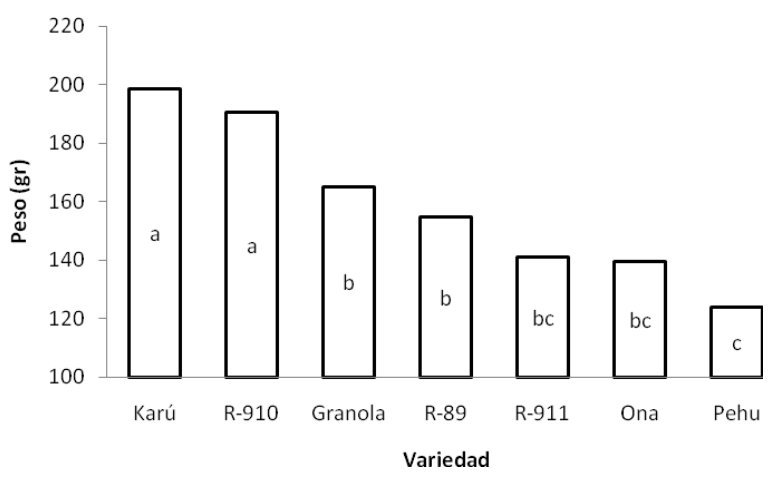

Figura 1. Peso promedio de los tubérculos en cada variedad. Diferentes letras indican diferencias significativas según la prueba de Rangos Múltiples de Duncan $(\mathrm{p}<0,01)$.

Se determinó que el contenido de sólidos totales en los tubérculos de las variedades Karú-INIA, R-91015-20 y Granola presentan los más bajos niveles $(<17 \%)$, mientras que Pehuenche-INIA, R-89054-3, R-91193-1 y Ona-INIA superan el $18 \%$ lo que indica su posible uso para procesamiento. Esta característica le añade un valor a la producción de estas variedades que, en casos puntuales, pudiera compensar los menores rendimientos.

En la Tabla 2 se presentan los rendimientos totales de las variedades para cada ambiente, así como la respectiva prueba de Duncan. El rendimiento promedio por ambiente varió desde $26.0 \mathrm{t} \cdot \mathrm{ha}^{-1}$ en Alto Pineda en época lluviosa, hasta $69.5 \mathrm{t} \cdot \mathrm{ha}^{-1}$ en Cerro Punta Centro (Anef) en época lluviosa. Esta diferencia entre los promedios de los ambientes refleja la variación entre los distintas condiciones edafoclimáticas a las cuales estuvieron sometidas las variedades. 
Tabla 2. Rendimiento total $\left(\mathrm{t} \cdot \mathrm{ha}^{-1}\right)$ de las variedades, por ambiente

\begin{tabular}{lccccccccc}
\hline \multirow{2}{*}{ Ambiente (Sitio) } & \multicolumn{7}{c}{ KENOTIPOS } \\
\cline { 2 - 7 } & $\begin{array}{l}\text { Kań } \\
\text { (G1) }\end{array}$ & $\begin{array}{c}\text { Ona } \\
(\mathrm{G} 2)\end{array}$ & $\begin{array}{c}\text { R-910 } \\
(\mathrm{G} 3)\end{array}$ & $\begin{array}{c}\text { Peh } \\
(\mathrm{G} 4)\end{array}$ & $\begin{array}{c}\text { R-911 } \\
(\mathrm{G} 5)\end{array}$ & $\begin{array}{c}\text { R-89 } \\
(\mathrm{G} 6)\end{array}$ & $\begin{array}{c}\text { Granola } \\
(\mathrm{G} 7)\end{array}$ & $\bar{x}$ \\
\hline Las Nubes (S1) & $39,2 \mathrm{a}$ & $36,7 \mathrm{~b}$ & $41,7 \mathrm{ab}$ & $56,5 \mathrm{a}$ & $35,3 \mathrm{~b}$ & $35,3 \mathrm{~b}$ & $41,8 \mathrm{a}$ & 40,93 \\
Alto Tribaldos (S2) & $28,1 \mathrm{~d}$ & $34,0 \mathrm{bc}$ & $27,9 \mathrm{~d}$ & $28,7 \mathrm{~cd}$ & $37,2 \mathrm{~b}$ & $13,7 \mathrm{e}$ & $47,2 \mathrm{a}$ & 30,97 \\
Alto Pineda (S3) & $28,5 \mathrm{ab}$ & $28,7 \mathrm{ab}$ & $24,4 \mathrm{bcd}$ & $33,7 \mathrm{a}$ & $20,8 \mathrm{~cd}$ & $19,0 \mathrm{~d}$ & $26,9 \mathrm{abc}$ & 26,00 \\
Anef, invierno (S4) & $79,8 \mathrm{a}$ & $64,6 \mathrm{bcd}$ & $74,1 \mathrm{ab}$ & $80,2 \mathrm{a}$ & $71,1 \mathrm{abc}$ & $57,5 \mathrm{~d}$ & $58,9 \mathrm{~cd}$ & 69,46 \\
Alto Las Nubes (S5) & $30,9 \mathrm{ab}$ & $22,8 \mathrm{~b}$ & $31,7 \mathrm{ab}$ & $39,6 \mathrm{a}$ & $20,4 \mathrm{~b}$ & $24,6 \mathrm{~b}$ & $30,7 \mathrm{ab}$ & 28,67 \\
La Garita (S6) & $28,9 \mathrm{bc}$ & $30,1 \mathrm{abc}$ & $17,6 \mathrm{~d}$ & $38,4 \mathrm{a}$ & $18,4 \mathrm{~d}$ & $22,5 \mathrm{~cd}$ & $31,5 \mathrm{ab}$ & 26,77 \\
Anef verano (S7) & $66,8 \mathrm{ab}$ & $60,5 \mathrm{~b}$ & $68,0 \mathrm{ab}$ & $72,9 \mathrm{a}$ & $68,1 \mathrm{ab}$ & $69,8 \mathrm{ab}$ & $76,3 \mathrm{a}$ & 68,91 \\
$\bar{x}$ & 43,17 & 39,63 & 40,77 & 50,00 & 38,76 & 34,63 & 44,76 & 41,69 \\
\hline
\end{tabular}

En la fila, las variedades seguidas de la misma letra no se diferencian estadísticamente entre sí, según la prueba de Rangos Múltiples de Duncan ( $<<0,01)$.

Se observa un mayor rendimiento promedio de todos los materiales en las localidades/ambientes de Anef invierno y verano (temporada lluviosa y seca respectivamente), lo que también refleja un alto nivel tecnológico de este productor.

En la Tabla 2 se presentan, además, los rendimientos promedio de cada uno de las variedades sobre todos los ambientes. Se registra un comportamiento distinto en los diversos ambientes, destacando en casi todas las localidades las variedades Pehuenche-INIA, Karú-INIA, Granola y R-91015-20. La adaptabilidad de variedades de papa para el cultivo convencional, donde todas las plantas tienen el mismo genotipo, en localidades y condiciones ambientales muy diferentes a las de su sitio de obtención, ha sido reportada por diversos autores (Estévez et al., 1999a, 1999b; Zamora et al., 1999). Por otro lado, R-89054-3 registró, en la mayoría de los ambientes, una producción significativamente inferior a las otras variedades, y una marcada incidencia de pudriciones de los tubérculos. El análisis de varianza, según el modelo AMMI para la variable rendimiento total de las variedades en siete ambientes detectó efectos altamente significativos $(\mathrm{p}<0,01)$ para las fuentes de variación: ambiente, genotipo y la interacción genotipo-ambiente (GxA), reiterando respectivamente las diferentes condiciones ambientales donde fueron evaluados los genotipos y un comportamiento relativo diferencial de los mismos al pasar de un ambiente a otro. El efecto principal del ambiente explicó el $80,4 \%$ de la suma de cuadrados total y el efecto principal del genotipo, solamente el $5,4 \%$, en tanto que la interacción genotipoambiente capturó el 7,8\%; esto indica que el modelo explica con precisión la interacción genotipo-ambiente de cada variedad en los ambientes evaluados (Cross et al., 1990). La mayor proporción en la suma de cuadrados para ambientes, en relación a la de genotipos, indica que los ambientes fueron contrastantes con marcadas diferencias entre las medias ambientales, ocasionando fluctuaciones en el rendimiento total. La magnitud de la suma de cuadrados de la interacción genotipo-ambiente, fue 1,4 veces mayor que la del genotipo, indicando que hubo diferencias significativas en la respuesta de los genotipos a través de los ambientes (Tabla 3). 
Tabla 3. Análisis AMMI para rendimiento de siete variedades de papa evaluados en siete ambientes. Panamá. IDIAP. Cerro Punta 2006-2007

\begin{tabular}{lcccc}
\hline Factor de Variación & Grados de libertad & Suma de Cuadrados & Cuadrados Medios & \% \\
\hline Ambiente (A) & 6 & 48030,062 & $8005,010^{* *}$ & 80,4 \\
Genotipo (G) & 6 & 3229,705 & $538,284^{* *}$ & 5,4 \\
G x A & 36 & 4676,950 & $129,915^{* *}$ & 7,8 \\
$\quad$-PCA1 & 11 & 3609,05 & $328,100^{* *}$ & 45,7 \\
$\quad$-PCA2 & 9 & 2033,69 & $225,966^{* *}$ & 25,7 \\
Error & 84 & 2902,62 & 34,555 & 4,9 \\
Total & 146 & 59707,856 & & \\
\hline
\end{tabular}

En la Figura 2 se presenta la gráfica Biplot GGE correspondiente a los dos primeros ejes del componente principal $\left(\mathrm{PCA}_{1}\right.$ y $\left.\mathrm{PCA}_{2}\right)$, para las siete variedades evaluadas en los siete ambientes, para la variable rendimiento total (ver Tabla 2 para los nombres completos de variedades y ambientes). Ambos ejes están explicando el $71,4 \%$ de la interacción genotipoambiente, el PCA1 explica el $45,7 \%$ de la interacción, mientras el PCA2, los restantes $25,7 \%$.
En la gráfica Biplot GGE, (Figura 2) los genotipos fueron representados por un punto, definido por el coeficiente de cada genotipo en el eje $\mathrm{PCA}_{1}$ contra el respectivo coeficiente en el eje $\mathrm{PCA}_{2}$ y los ambientes fueron representados como vectores con origen en el punto 0,0 , extendiéndose hasta el marcador respectivo. Mientras más cerca, del centro de la gráfica, se localiza un genotipo, menos responde a las variaciones ambientales.

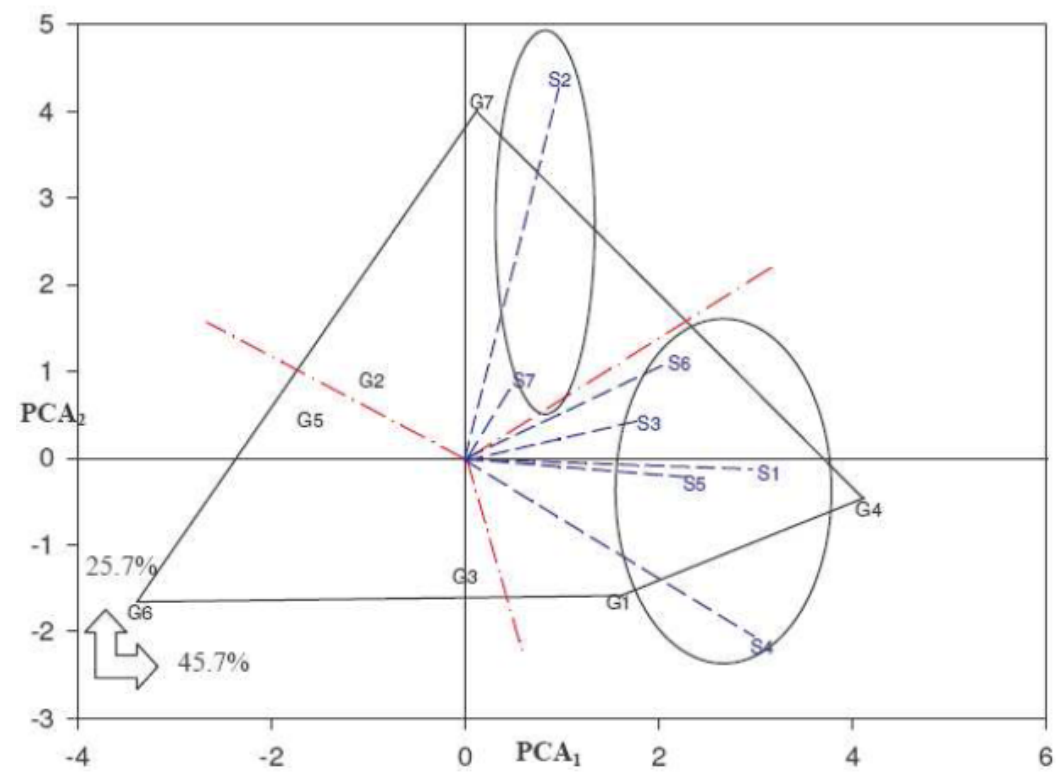

Figura 2. Puntuaciones del primer y segundo ejes del componente principal de siete cultivares de papa en siete localidades. IDIAP. Cerro Punta, Panamá. 2005-2007 
Entre tanto, aquellos ubicados en los vértices o esquinas del polígono son considerados los que más aportan a la interacción es decir que más responden a los estímulos ambientales. Esta respuesta es positiva ó negativa, dependiendo del signo y la magnitud de la puntuación. Observamos, en los vértices, respondiendo positivamente a los estímulos ambientales, a las siguientes variedades: Karú-INIA (G1), Pehuenche-INIA (G4), Granola (G7) y R-9101520(G3). Por el contrario, los genotipos R-890543(G6), R-91193-1 (G5) y Ona-INIA (G2) respondieron de forma negativa.

La variedad Pehuenche-INIA, fue identificada por el modelo como el más estable en su rendimiento y además, con la mejor adaptabilidad. La estabilidad en este modelo está asociada a valores del PCA2 cercanas a cero y las variedades Pehuenche-INIA, Karú-INIA (G1) y R-91193-1 (G5) fueron las que presentaron los valores más cercanos a cero, con la diferencia de que la primera mostró los más altos valores del PCA1, lo que indica una mejor adaptabilidad, expresada en este caso, con altos rendimientos a través de las localidades. La ubicación de las variedades Karú-INIA y Pehuenche-INIA en vecindad con las localidades Alto Pineda, Anef Invierno, Las Nubes, La Garita y Alto Las Nubes, indica que estos representaron los ambientes más favorables para dichas variedades (Yan et al., 2000). Granola correlacionó más estrechamente con las localidades Alto Tribaldo y Anef verano; esta variedad, que se ha sostenido en la producción por muchos años, en este estudio, mostró rendimientos ligeramente superiores al promedio general, sin embargo, por su alto valor del $\mathrm{PCA}_{1}$ se deduce que es sensible a los estímulos ambientales.

Las variedades R-89054-3, R-91193-1 y OnaINIA, presentaron el comportamiento más pobre en la mayoría de los ambientes, lo que es apreciable por su ubicación alejada de los sectores donde se encuentran los vectores de los ambientes. En este caso hay una correlación negativa entre genotipos y ambientes.

La figura nos permite identificar las localidades Alto Tribaldo y Anef invierno como las de mayor capacidad discriminadora para este grupo de genotipos, lo que queda de manifiesto por la mayor longitud de los vectores de estas localidades.

\section{Conclusiones}

* La variedad Pehuenche-INIA, es promisoria para productores de papa de Panamá, por su alto potencial de rendimiento, estabilidad y alto contenido de sólidos en los tubérculos.

* La variedad Karú-INIA y el clon R-9101520 son aptos para consumo fresco y representan alternativas para productores de papa de Panamá, por su alto potencial de rendimiento.

* Las variedades Karú-INIA y R-91015-20 se caracterizan por tubérculos grandes $\mathrm{y}$ Pehuenche-INIA, por tubérculos de menor tamaño.

\section{Agradecimientos}

A la Secretaría Nacional de Ciencia, Tecnología e Innovación de Panamá (SENACYT), que financió parcialmente la ejecución del proyecto "Desarrollo de la industria de procesamiento de papa en Panamá" del cual es parte el presente estudio.

\section{Literatura Citada}

Bradshaw, J.E., MacKay, G.R. 1994. Breeding strategies for clonally propagated potatoes. En: Potato Genetics. Wallingford, U.K. Center for Agriculture and Bioscience International Publishing. pp. 467-498.

Centro Internacional de la Papa (CIP). 2007. Procedures for standard evaluation trials of advanced potato clones. An International Cooperators' Guide. Lima, Peru. 127 p.

Contreras, S., Krarup, C. 2000. Interacción genotipo por ambiente en cinco cultivares de espárrago (Asparragus officinalis L.). Ciencia e Investigación Agraria (Chile) 27:133-139.

Cornelius, P.L., Crossa, J., Seyedsader, M.S. 1996. Statistical tests and estimators of multiplicative models for genotype-by-environment interaction. In M.S. Kang and H.G. Gauch (ed.) Genotype-by- 
environment interaction. CRC Press, Boca Raton, FL. pp. 199-234.

Cross, J., Gauch, H., Zobel, R. 1990. Additive main effects and multiplicative interaccion of two international maize cultivar trials. Crop Science 30:493-500.

Crossa, J. 1990. Statistical analyses of multilocations trials. Edited by: N.C. Brady In: Advances in Agronomy 44:55-85. Academic Press. ISBN: 9780120007448.

Crossa, J., Cornelius, P.L. 1997. Sites regression and shifted multiplicative model clustering of cultivar trial sites under heterogeneity of error variances. Crop Science 37: 405-415.

Dale, M.F., MacKay, G.R. 1994. Inheritance of table and processing quality. In: Potato Genetics. Wallingford, U.K. Center for Agriculture and Bioscience International Publishing. pp. 285-315.

Estévez, A., González, M.E., Castillo, J., Ortiz, E. 1999a. Estudio de genotipos de papa procedentes del germoplasma foráneo. Compendio de exposiciones del II Taller Nacional de Producción de papas en los Trópicos. La Habana. pp. 57-58.

Estévez, A., González, M.E., Castillo, J. 1999b. Evaluación y selección de clones cubanos de papa de diferentes generaciones. Compendio del III taller de producción de papa en los trópicos. La Habana. pp. 68-70.

Instituto de Investigación Agropecuaria de Panamá (IDIAP). 1984. Cerro Totuma, Cerro Picacho y Cerro Punta. Idiapuntes. Edición especial. No 3 y 4 . pp. 2-5.

Kumlay, A.M., Kaya, C., Olgun, M., Dursun, A., Pehluvan, M., Dizikisa, T. 2002. Comparison of seasonal change of specific gravity, dry matter accumulation and starch content of four potato (solanum tuberosum 1.) varieties. En: Acta Hort. (ISHS) 579:255-258. http://www.actahort.org/books/579/579 42.htm . Consulta junio 2006.
Ministerio de Desarrollo Agropecuario de Panamá (MIDA). 2006. Dirección de planificación sectorial. Análisis de consumo aparente de papa. $1 \mathrm{p}$.

Scapim, C.A., Oliveira, V.R., Braccini, A.L., Cruz, C.D., Andrade, C.A.B., Vidigal, M.C.G. 2000. Yield stability in maize (Zea mays L.) and correlations among the parameters of the Eberhart and Russell, Lin and Binns and Huehn models. Genet. Mol. Biol. 23:387-393.

Vargas, M., Crossa, J. 2000. El análisis AMMI y la grafica del biplot en SAS. Unidad de biometría y estadística, CIMMYT. México. 42 p.

Westermann, D.T., Tindall, T.A., James, D.W., Hurst, R.L. 1994. Nitrogen and potassium fertilization of potatoes: Yield and specific gravity. American Potato Journal 71:417-431.

Yan, W., Hunt, L., Sheng, Q., Szlavnics Z. 2000. Cultivar Evaluation and Mega Environment Investigation based on the GGE Biplot. Crop Science 40:597-605.

Yue, G.L., Roozeboom, K.L., Schapaugh, W.T., Liang, G.H. 1997. Evaluation of soybean cultivars using parametric and nonparametric stability estimates. Plant Breeding 116: 271-275.

Zamora, N., Salomón, J., Estévez, A., González, M.E., Cordero, M., López, E., Miranda, R. 1999. Selección de variedades foráneas de papa (Solanum tuberosum, L.) en las condiciones de Cuba. Compendio de exposiciones del III Taller de Producción de papa en los trópicos. La Habana. pp. 82-83.

Zobel, W., Wright, M., Gauch, H.G. 1988. Statistical analysis of a yield trial. Agron J. 80:388-393. 